\title{
Research Paper: Internet Addiction in High School Students and Its Relationship With the Symptoms of Mental Disorders
}

Parvaneh Mohammadkhani ${ }^{1}$, Emad Alkasir ${ }^{*}$, Abbas Pourshahbaz ${ }^{1}$, Fatemeh Jafarian Dehkordi ${ }^{2}$, Erfan Soleimani Sefat ${ }^{3}$

1. Department of Clinical Psychology, University of Social Welfare and Rehabilitation Sciences, Tehran, Iran.

2. Faculty of Education and Psychology, University of Mohaghegh Ardabili, Iran

3. Department of Counseling, University of Social Welfare and Rehabilitation Sciences, Tehran, Iran.

Article info:

Received: 08 Nov. 2016

Accepted: 12 Mar. 2017

Keywords:

Internet addiction, Mental disorder, High school students

\begin{abstract}
Objectives: One of the problems that the Internet has created is a virtual addiction or addiction to the global web world causing adverse psychological behavioral effects on the users. One of the groups at higher risk for Internet addiction is teenagers, and since Internet addiction will lead to academic failure in students, evaluating it among them and its relationship with signs of mental disorders seems necessary. Therefore, the aim of this research was to study Internet addiction and its relationship with the symptoms of mental disorders
\end{abstract}

Methods: This study was descriptive and correlational design. The sample population for the present study consisted of all male and female high school students of the academic year 2013-2014 in district 5 of Tehran. Among these individuals, a sample of 400 participants was randomly selected for analysis using cluster sampling method. To collect data, Internet addiction test and Brief Symptom Inventory were used, and data were analyzed using T-Test, Pearson correlation coefficient and multiple regression analysis.

Results: The results showed that there is no significant difference between prevalence of Internet addiction in male and female high school students in district 5 of Tehran. However, there is a significant positive relationship between Internet addiction variables and symptoms of mental disorders and particularly, independent variables of psychosis and anxiety from the signs of mental disorders had a profound effect on Internet addiction.

Discussion: Internet addiction causes negative effects on individuals. As prevention is better than treatment and according to this study, it is necessary to take this phenomenon into consideration as a psychological problem that often involves the younger generation who are responsible for future society construction and through education in families, schools and universities, the culture of proper use of computers, in particular the Internet and its facilities should be replaced with the false methods.

* Corresponding Author:

Emad Alkasir, $M A$

Address: Department of Clinical Psychology, University of Social Welfare and Rehabilitation Sciences, Tehran, Iran.

Tel: +98 (916) 8047848

E-mail: emadalkasir@yahoo.com 


\section{Introduction}

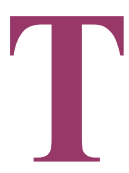

he revolution in computer technology and communication through the Internet play a pivotal role in today's culture and has affected individuals of different ages. In the present scenario, Internet and computer technology are considered as the most effective tool and has been influential in all areas of science, business, education, culture, politics, etc. [1].

One of the major problems that the Internet has created is virtual addiction or addiction to the global web world, which has harmful psychological and behavioral effects on the user [2]. Nowadays, Internet addiction has been raised as a mental disorder in psychology and medical science and this disorder as a new form of addiction in recent years has attracted the attention of researchers from various fields including psychology-sociology, psychiatry, sociology and other disciplines $[3,4]$.

The international prevalence of $1.5 \%$ to $8.2 \%$ has been reported for Internet addiction [5]. In Iran, National Youth Organization research analysis indicates that the number of Internet users in Iran, with a growth of about $90 \% 7.1$ million people with a penetration rate of $2 / 5 \%$ in 2000 has increased to 3.2 million with a penetration rate of $4.9 \%$ by the end of 2011 [6]. It is anticipated that due to the increasing Internet penetration in Iran, the number of Internet users in schools and universities will reach to 15 million per day. Based on these statistics, more than $35 \%$ of Internet users are young people. The statistical report shows that the number of Internet users in Iran has grown up to 25 times [7].

Several studies indicate that the number of students using the Internet and the incidence and prevalence of Internet addiction among them is increasing [8]. In Greece, Internet addiction was $11 \%$ in this age group; in South Korea, it was 7-10\% [9]; in Finland, it was 7.4\% in females and $6.4 \%$ in males [10]; in China, it was 9 to $11 \%$ [11]; and in Italy, $4.5 \%$ of students were addicted to the Internet [12]. There has been limited research on the prevalence of Internet addiction in high school students in Iran. The Ghasemzadeh and colleagues' study [13] on the prevalence of Internet addiction in Iran is one of the few researches in this field. In Iran, the Internet addiction prevalence rate among high school girls is estimated to be about $8.3 \%[13]$.

Addiction to the Internet varies among different societies and different age populations. One of the groups that are at higher risk for Internet addiction is adoles- cents. Due to educational, information, entertainment needs and for other requirements, students tend to use more Internet. Since they have more free time to use the Internet, they are more susceptible to the phenomenon of "Internet addiction" [14, 15]. Moreover, given that Internet addiction is associated with various disorders, which leads to poor performance in school and society, it seems essential to investigate the relationship of Internet addiction with psychological disorders symptoms carefully in students.

In addition to the suffering and limitations that the mental disorder creates in the individual, it also causes the person to suffer from discriminations in his/her social and professional activities because of the stigma of mental illness. The impact of mental disorders on society is very important due to their need for care and physical support and their loss of productivity. The burden of medical expenses and physical and emotional care and support for patients' families are burdened. Moreover, there is an economic burden, which should not be ignored [16]. Internet addiction in students is associated with problems such as loss of interpersonal communication, anger, aggression and irritability [9].

It can also cause multiple harms to the person, including mental health (anxiety, depression, and loneliness), physical (sleep disorders, nutrition), familial (reduction of family relationships and increasing the distance between family members), job (reduced work effectiveness, dismissal from work) and education (school grades drop) [17]. Internet addiction in students is concomitant with depression and insomnia [18], suicidal thoughts [19], hyperactivity disorder (ADHD), social phobia and hostility [20], drug abuse [21], and problematic alcohol consumption [22]. A total of 78\% of German students who were addicted to the Internet had depression and impulsivity disorder [23].

There was co-morbidity with mood disorder obsessive compulsive personality disorder while borderline personality disorder was observed in American students who were addicted to the Internet [12]. A combination of Alexitimia, dissociative experiences low self-esteem and impulsivity disorder were identified as risk factors of addiction to the Internet in Italian adolescents [24]. Adolescents who were addicted to the Internet had more signs of hyperactivity, depression, social phobia and hostility in Taiwan [14].

According to the findings of Bahri et al [25], there is a significant and negative statistical relationship between addiction to the Internet and general health. Another 
study by Alavi et al. [3] indicated that men are 1.8 times more at risk of addiction to the Internet than women, and there is a significant and positive statistical relationship between scores of psychiatric signs (such as depression, anxiety, hypochondria, obsessive compulsive disorder, interpersonal sensitivity, aggression, paranoia, phobia and psychosis) and addiction to the Internet.

Nastizayi [26] indicated that general health of addicted Internet users, especially in subscales of anxiety and depression users, is at higher risk as compared with normal users. Another study by Bidi et al. [27] shows that there is a positive and significant relationship between depression and addiction to the Internet. The results of a study by Akin and Iskender [28] showed that Internet addiction has a direct and significant relationship with stress, depression, and anxiety. The results of a study by Kim and colleagues [29] shows that $1.6 \%$ of these people have Internet addiction, and 38\% of them were at risk of Internet addiction; the level of depression and suicide has been reported in the group of people addicted to the Internet.

Results of the -Wan oh study showed that $27 \%$ of students were addicted or were at a high risk of Internet addiction. The study also showed that there is a positive correlation between Internet addiction and depression [30]. The results of a study by Masoudnia [31] shows the extreme pathological use of the Internet is a strong risk factor for the teens' sleep quality and increases the risk of sleep disorders in adolescents. Janvcha et al. [32] have acknowledged that the majority of people susceptible to damage are students and young people who must be considered for treatment, and therefore, the prevalence of mental disorders in adolescents and its relationship with the symptoms will be discussed.

\section{Methods}

In this study, the causal-comparative method has been used. The type of research is descriptive - correlation. Research population constitutes of all high school students, both girls and boys of district 5 in Tehran and who were studying in 2013-14 academic year. The statistical population reported is 8460 boys and 7840 girls. In this study, to calculate the sample, Cochran formula was used by which the sample size of 400 was calculated. In this study Cluster sampling was used for the criterion of gender.

\section{Inclusion criteria}

The individuals who participated in the study as subjects had the following criteria: 1 . High school student of District 5 in Tehran. 2. High school students from first to fourth grade.

The standardized Internet addiction questionnaire that was built in 1998 by Kimberly Yang was used. The questionnaire included 20 items on a 5-point Likert scale from zero (never) to score four (always) were measured among students. In a study by Alavi et al., Cronbach's alpha coefficient for the total scale was equal to $88 \%$ that for the five factors to $a=81 \%, a=73 \%, a=75 \%, a=74 \%$, $\mathrm{a}=61 \%$. To determine the validity of questionnaires, $\mathrm{Bi}$ section Method was used. In this method, by using the odd and even scores of the questions, the correlation coefficient of $82 \%$ was obtained $(\mathrm{P}>0.01)$.

\section{Brief Symptom Inventory (BSI)}

Brief Symptom Inventory is the short form of SCL-90-R [33] and a means of screening psychological disorders. This questionnaire included the following nine symptoms: somatization (SOM), obsessive-compulsive (OC), interpersonal sensitivity (IS), depression (DEP), anxiety (AX), hostility (HOS), phobic anxiety (PHOB), paranoia (PAR), and psychoticism (PSY). The questionnaire also has three overall indexes: the total Positive Symptoms (PST), (the number of psychiatric symptoms regardless of the severity of each measure), Positive Symptom Distress index to assess the severity of symptoms, and Global Severity Index PSDI (GSI) (the scores indicate the number and severity of symptoms of distress).

Each phrase of the questionnaire measures turmoil in a 5-point scale from 0 "not at all" to 4-severe". Although $\mathrm{BSI}$ is designed to measure psychiatric symptoms, Factor structure of its basic form is shown by a number of studies. Deragotis 9 Operating considers and states that although a slight difference between the practical and the structure of dimensions are given; more consensus than differences between the two versions is observed. This questionnaire is a useful tool for researchers, particularly working related to the results and assessment of clinical treatment [34].

\section{Data analysis method}

Data obtained from the study using Statistical Package for Social Sciences (SPSS, v.22) were further analyzed. The values used descriptive statistics to summarize demographic characteristics. Independent T-Tests for continuous variables and chi-square tests were used in order to assess the statistical significance of any differences and relationships between parameters of the research in question. Pearson correlation coefficient for calculating 
correlations Stepwise multiple regression and two standard methods mentioned variables were calculated to assess the predictive power. For all evaluations, $\mathrm{P}<0.5$ was considered as a significant difference between the two sides.

\section{Results}

In this research, the age of users ranged from 15 to 18 years, and the average age was 16 years. The gender distribution of the sample population showed equal proportion of boys and girls. The findings also showed that the educational level in the study sample was $56.3 \%(n=225)$ freshman of high school, $32.8 \%(n=131)$ sophomore of high school and $11 \%(n=44)$ were junior of high school.

The findings also showed that the academic performance of the sample, $10 \%(\mathrm{n}=40)$ with grade point average of $14-12,33.8 \%$ (135) have a grade point average between $17-15$, and $56.3 \%$ (225 patients) had grade point average of 20-18. According to Table 1 and T-Test results, $\mathrm{F}=0.950$ and $\mathrm{Sig}=0.758$, there is no significant difference between the prevalence of Internet addiction in male and female students in the secondary district 5 of Tehran.

According to Pearson correlation coefficient as shown in Table 2, there is a significant positive relationship between Internet addiction and variables of mental disorder and somatization, including obsessive-compulsive, interpersonal sensitivity, depression, anxiety, hostility, phobic anxiety, paranoid ideation and psychoticism (Table 3).

Table 4 deals with the significance level of the regression model. Given that the predictor variables and criteria were based on the distance scale, and a linear relationship was observed between them, and normal distribution of data, naturalness and consistency remaining dispersive were screened, so there are regression assumptions.

According to Table 5, the effects of each component of somatization, obsessive-compulsive, interpersonal sensitivity, depression, anxiety, hostility, phobic anxiety, paranoid ideation and psychoticism as predictor vari-

Table 1. Independent t-tests of internet addiction variable among girls and boys

\begin{tabular}{|c|c|c|c|c|c|c|c|}
\hline \multirow{2}{*}{ Variable } & \multicolumn{2}{|c|}{ Levine Test } & \multirow{2}{*}{$\mathbf{t}$} & \multirow{2}{*}{ df } & \multirow{2}{*}{ MD } & \multirow{2}{*}{ Minimum } & \multirow{2}{*}{ Maximum } \\
\hline & $\mathbf{F}$ & Sig. & & & & & \\
\hline Internet addiction & 0.095 & 0.758 & 2.764 & 398 & 4.1 & 1.19 & 7 \\
\hline
\end{tabular}

Table 2. Pearson correlation between symptoms of mental disorders and its components with Internet addiction

\begin{tabular}{|c|c|c|c|c|c|c|c|c|c|c|c|c|}
\hline & Variables & 1 & 2 & 3 & 4 & 5 & 6 & 7 & 8 & 9 & 10 & 11 \\
\hline 1 & Internet addiction & 1 & & & & & & & & & & \\
\hline 2 & BSI & $* 0.28$ & 1 & & & & & & & & & \\
\hline 3 & Physical complaints & $* 0.26$ & $* 0.82$ & 1 & & & & & & & & \\
\hline 4 & Obsessive compulsion & $* 0.28$ & $* 0.87$ & *0.69 & 1 & & & & & & & \\
\hline 5 & $\begin{array}{l}\text { Sensitivity in interpersonal } \\
\text { relations }\end{array}$ & $* 0.17$ & $* 0.83$ & $* 0.60$ & $* 0.70$ & 1 & & & & & & \\
\hline 6 & Depression & $* 0.20$ & $* 0.89$ & $* 0.65$ & ${ }^{*} 0.75$ & $* 0.73$ & 1 & & & & & \\
\hline 7 & Anxiety & $* 0.25$ & $* 0.86$ & $* 0.75$ & $* 0.69$ & $* 0.66$ & $* 0.72$ & 1 & & & & \\
\hline 8 & Aggression & $* 0.29$ & $* 0.85$ & $* 0.73$ & $* 0.74$ & $* 0.65$ & $* 0.70$ & $* 0.73$ & 1 & & & \\
\hline 9 & Phobic anxiety & $* 0.20$ & $* 0.76$ & $* 0.63$ & $* 0.60$ & $* 0.58$ & $* 0.60$ & $* 0.66$ & $* 0.62$ & 1 & & \\
\hline 10 & Paranoid ideation & $* 0.24$ & $* 0.82$ & $* 0.55$ & $* 0.66$ & $* 0.70$ & $* 0.76$ & $* 0.66$ & $* 0.62$ & $* 0.53$ & 1 & \\
\hline 11 & Psychosis & ${ }^{*} 0.23$ & ${ }^{*} 0.87$ & ${ }^{*} 0.65$ & $* 0.75$ & $* 0.71$ & $* 0.79$ & ${ }^{*} 0.71$ & ${ }^{*} 0.71$ & ${ }^{*} 0.69$ & ${ }^{*} 0.69$ & 1 \\
\hline
\end{tabular}


Table 3. Correlation coefficients table predictors of symptoms of mental disorders

\begin{tabular}{ccccc}
\hline Model & Correlation Coefficients & $\mathbf{R}^{2}$ Adjusted & Standard Error & Durbin-Watson \\
\hline 1 & ${ }^{\mathrm{a}} 0.456$ & 0.298 & 12.8 & 2.01 \\
\hline Predictors: Symptoms of mental disorders & & & $\underline{\text { ॥ranian Rehabilitation』ournal }}$
\end{tabular}

Variable: Internet addiction

Table 4. Significant results of regression (ANOVA)

\begin{tabular}{cccccc}
\hline Model & SS & df & MS & F & Sig. \\
\hline Regression & 27226.9 & 9 & 3025.21 & & 0.000 \\
Remaining & 63994.03 & 390 & & 164.08 & \\
Total & 91220.93 & 399 & & \\
\hline
\end{tabular}

ables and Internet addiction as dependent variable were analyzed by multivariate regression analysis. According to the results, it can be concluded that only independent variable of the study psychoticism and anxiety are effective on Internet Addiction. The psychopathic-oriented vary according to the coefficient of determination $(B=0.187)$ and anxiety $(B=0.156)$ indicate that Internet addiction in male and female are high school students.

\section{Discussion}

According to the results of this research, the prevalence of Internet addiction in male and female students did not show any significant difference. This finding is also consistent with the results of the investigation by Moham- madi et al. [35] and Tamanaeifar et al. [36] indicating that there is no significant difference in the prevalence of Internet addiction among girls and boys. While explaining these results, in developed societies and classes where the Internet is more incredibly accessed and there is no significant difference between boys and girls, the factors of culture and the region could have a huge impact on Internet addiction.

Other results also showed that there is a significant relationship between symptoms of mental disorders and its components and Internet addiction in high school students. Furthermore, there is a significant positive relationship between the variables of Internet addiction and psychiatric disorder and somatization,

Table 5. Table variables entered into the regression equation and coefficients

\begin{tabular}{|ccccc}
\hline Variables & $\mathbf{R}^{2}$ Not Adjusted & Beta & T & Sig. \\
\hline Constant & 10.18 & -- & 8.7 & 0.00 \\
\hline Physical complaints & 0.042 & 0.015 & 0.20 & 0.84 \\
\hline Obsessive-compulsion & 0.143 & 0.047 & 0.60 & 0.54 \\
\hline Sensitivity in interpersonal relations & -0.30 & -0.073 & -1.02 & 0.30 \\
\hline Depression & 0.186 & 0.075 & 0.87 & 0.38 \\
\hline Anxiety & 0.51 & 0.156 & 1.99 & 0.047 \\
\hline Aggression & 0.48 & 0.13 & 1.72 & 0.085 \\
\hline Phobic anxiety & 0.15 & 0.037 & 0.58 & 0.55 \\
\hline Paranoid ideation & 0.12 & 0.040 & 0.56 & 0.57 \\
\hline Psychosis & 0.67 & 0.187 & 2.25 & 0.025 \\
\hline
\end{tabular}


obsessive-compulsive, interpersonal sensitivity, depression, anxiety, hostility, phobic anxiety, paranoid ideation and psychoticism, which is consistent with the results of several earlier studies. For example, a study by Masoudnia [31] showed that Internet addiction in adolescents increases the risk of sleep disorders. Likewise, Shari et al. [37] showed that general health status, and anxiety and depression in students addicted to the Internet is significantly worse.

In another study by Alavi et al. [3], it was found that there is a significant positive correlation among mental disorders such as depression, anxiety, hypochondriasis, obsessive-compulsive, interpersonal sensitivity, hostility, paranoia, phobias and psychotic with Internet addiction. The findings of Nastizai [26] showed that the health of people who are addicted to Internet users especially in the domains of anxiety and depression are at greater risk than ordinary users. Similarly, in another study by Bidi and colleagues [27], it was showed that depression in a positively and meaningful way is correlated with Internet addiction.

The research results of Eakin et al. [28] showed that Internet addiction has a direct and significant relationship with stress, depression and anxiety. A study by Kim et al. [29] showed that the level of depression and suicide in the group who are addicted to the Internet is high. Likewise, another study by Martin and Schumacher [38] also concluded that Internet addicted users as compared to those who had no signs of dependence on Internet, were relatively more lonelier and depressed. In explaining these results, the Internet addicted individuals were observed to have less willingness to communicate and relate in fact, the extreme dependence of the virtual world with detachment from reality and physical inactivity in the associated person, reduces happiness and social interactions.

As a result, the user's mind engages with imagination inefficiently leading to depression, anxiety and social isolation, and in general with no mental disorders. On the other hand, addicted users have considerable anxiety and apprehension. These people might have used Internet as a loophole, and this probably indicates that when a person does not have access to the Internet get anxious and to reduce their anxiety uses Internet [39]. Based on the findings, people who excessively use the Internet, become distant from the community and actual social communications and by eliminating the real social interaction and control over their lives, they can be associated with loneliness and depression. Since users often online appointment contrived and weak ties and preferred objective of the interactions of life, real life relationships are less valued and the same can be associated with depression.

With increasing depression and loneliness, the internet addict becomes connected to the Internet for longer periods. The same observation can also be seen in an alcoholic, who drinks to escape from problems, then, feeling worse ultimately drinks more to get rid of unpleasant emotions. The results obtained in this debate, Proof of principle of co-morbidity with other disorders is Internet addiction. Internet addiction is associated with another disorder that is often physical and mental.

According to the results it can be said that the only independent variable of research psychoticism and anxiety are affecting Internet addiction, and which is consistent with the results of a previous study by Won Oh et al. [30] on propensity to determine the risk factors for Internet addiction which showed that there is a negative correlation between Internet addiction and relationship with personal connections, family support and self-control while there is a positive relationship between Internet addiction and depression. Likewise, in another study by Cui et al. [11] suggest that Internet addiction is associated with alcohol abuse, ADHD, depression and anxiety, particularly $26.3 \%$ of patients with Internet addiction suffer from depression, $21.7 \%$ of ADHD and $23.3 \%$ of anxiety.

Regression analysis also suggests that there is a positive relationship between Internet addiction and a series of clinical variables and the clinical variables can predict $39 \%$ of Internet addiction. Jafari and Fatehizadeh [40] have also showed that there is a significant positive relationship between Internet addiction and clinical variables of depression, anxiety, stress and social phobia in concomitant with the findings of the Alavi et al. [3] who stated that there is a significant positive correlation between the scores of psychiatric symptoms (such as depression, anxiety, hypochondriasis, obsessive-compulsive, interpersonal sensitivity, aggressiveness, paranoia phobias and psychotic) and Internet addiction.

It can be said that individuals who are psychotic and anxious due to their disorder are more inclined to refrain from others, and remain in isolation and tranquility which attracts them to the mass media, including TV and computer for consuming a lot of their leisure time and thereby begin to suffer from Internet addiction. They also have a feeling of insecurity, loneliness and lack of control over the environment and lack of social support networks that this tendency platform provides them to the Internet why via the Internet loneliness and lack of control over the environment and reduce the lack of support networks. 


\section{Limitations}

The present study was a cross-sectional research and analysis on Internet addiction, personality traits and psychiatric symptoms. However, it requires more precise longitudinal studies to confirm the causal relationships between Internet addiction, personality traits and psychiatric symptoms. Due to the use of self-measurement scales, the individual's response may be exaggerated by their interest in self-presentation or self-expression. As a result some data and results observed in this study might have been affected by people's opinions.

\section{Conclusion}

Internet addiction leaves negative effects on individuals. As prevention is better than treatment and according to this study it is necessary to take this phenomenon into consideration as a psychological problem that often involves the younger generation who are responsible for future society construction and through education in families, schools and universities, the culture of proper use of computers, in particular the Internet and its facilities should be replaced with the false methods.

\section{Acknowledgments}

The authors would like to express their appreciation to the students who participated in this study. It is hoped that this research has provided a further step for attracting attentions of individuals, research institutions, and government organizations towards both male and female students. This article is taken from the second author master's thesis titled "The prevalence of Internet addiction and its relationship with personality charactristics and psychiatric symptoms in highschool students."

\section{Conflict of Interest}

The authors declared no conflicts of interest.

\section{Refrences}

[1] Brenner V. Psychology of computer use: XLVII, parameters of Internet use, abuse and addiction: The first 90 days of the Internet usage survey. Psychological Reports. 1997; 80(3):879_ 82. doi: $10.2466 /$ pr0.1997.80.3.879

[2] Widyanto L, Griffiths M. Internet Addiction: A critical review. International Journal of Mental Health and Addiction. 2006; 4(1):31-51. doi: 10.1007/s11469-006-9009-9
[3] Alavi SS, Jannatifard F, Maracy M, Rezapour H. [The psychometric properties generalized pathological internet use scale (GPIUS) in Internet users students of Isfahan Universities (Persian)]. Journal of Knowledge \& Research in Applied Psychology. 2009; (40):38-51.

[4] Stavropoulos V, Alexandraki K, Motti-Stefanidi F. Recognizing internet addiction: Prevalence and relationship to academic achievement in adolescents enrolled in urban and rural Greek high schools. Journal of Adolescence. 2013; 36(3):56576. doi: 10.1016/j.adolescence.2013.03.008

[5] Petersen K, Weymann N, Schelb Y, Thiel R, Thomasius R. [Pathological Internet use--epidemiology, diagnostics, co-occurring disorders and treatment (German)]. Fortschritte der Neurologie Psychiatrie. 2009; 77(05):263-71. doi: 10.1055/s0028-1109361

[6] Navabakhsh M, Hashem neghad F, Zadeshampour V. [A study of Internet and cell phone effects on the youth (15-29 years of old) and identity change in Mazandaran (Persian)]. Sociological Studies of Youth Journal. 2006; 1(1):145-70.

[7] Shahghassemy A. [Review on effect of virtual space fields on communication theories (Persian)]. Global Media JournalPersian Edition. 2006; 1(2):31-7.

[8] Siomos KE, Dafouli ED, Braimiotis DA, Mouzas OD, Angelopoulos NV. Internet addiction among Greek adolescent students. Cyber Psychology \& Behavior. 2008; 11(6):653-7. doi: $10.1089 / \mathrm{cpb} .2008 .0088$

[9] Park SK, Kim JY, Cho CB. Prevalence of internet addiction and correlations with family factors among South Korean adolescents. Adolescence. 2008; 43(172):895. PMID: 19149152

[10] Cooper A. Sex and the internet: A guidebook for clinicians. New York: Psychology Press; 2002.

[11] Lijuan C, Xin Z, Mingzheng W. A research on the effects of Internet addiction on adolescents' social development. Psychological Science-Shanghai. 2006; 29(1):34.

[12] Pallanti S, Bernardi S, Quercioli L. The shorter PROMIS questionnaire and the internet addiction scale in the assessment of multiple addictions in a high-school population: Prevalence and related disability. CNS Spectrums. 2006; 11(12):966-74. doi: 10.1017/s1092852900015157

[13] Ghasemzadeh L, Shahrarai M, Moradi A. [The extent of internet addiction and its relation to loneliness and self esteem among Tehran high school students (Persian)]. Quarterly Journal of Education. 2007; 23(1):41-68.

[14] Yen JY, Ko CH, Yen CF, Wu HY, Yang MJ. The comorbid psychiatric symptoms of internet addiction: Attention deficit and hyperactivity disorder (ADHD), depression, social phobia, and hostility. Journal of Adolescent Health . 2007; 41(1):93-8. doi: 10.1016/j.jadohealth.2007.02.002

[15] Cumbie SA, Conley VM, Burman ME. Advanced practice nursing model for comprehensive care with chronic illness. Advances in Nursing Science. 2004; 27(1):70-80. doi: 10.1097/00012272-200401000-00008

[16] Sadock BJ, Sadock VA. Kaplan and sadock's synopsis of psychiatry: Behavioral sciences/clinical psychiatry. Pennsylvania: Lippincott Williams \& Wilkins; 2011. 
[17] Flisher C. Getting plugged in: An overview of internet addiction. Journal of Paediatrics and Child Health. 2010 46(10):557-9. doi: 10.1111/j.1440-1754.2010.01879.x

[18] Ceung LM, Wong WS. The effects of insomnia and internet addiction on depression in Hong Kong Chinese adolescents: An exploratory cross-sectional analysis. Journal of Sleep Research. 2010; 20(2):311-7. doi: 10.1111/j.13652869.2010.00883.x

[19] Fu K w., Chan WSC, Wong PWC, Yip PSF. Internet addiction: prevalence, discriminant validity and correlates among adolescents in Hong Kong. The British Journal of Psychiatry. 2010; 196(6):486-92. doi: 10.1192/bjp.bp.109.075002

[20] Ko CH, Yen JY, Chen CS, Yeh YC, Yen CF. Predictive values of psychiatric symptoms for internet addiction in adolescents. Archives of Pediatrics \& Adolescent Medicine. 2009; 163(10):937. doi: 10.1001/archpediatrics.2009.159

[21] Gong J, Chen X, Zeng J, Li F, Zhou D, Wang Z. Adolescent addictive internet use and drug abuse in Wuhan, China. Addiction Research \& Theory. 2009; 17(3):291-305. doi: $10.1080 / 16066350802435152$

[22] Ko CH, Yen JY, Chen CS, Chen CC, Yen CF. Psychiatric comorbidity of internet addiction in college students: An interview study. CNS Spectrums. 2008; 13(02):147-53. doi: $10.1017 /$ s1092852900016308

[23] Te Wildt B, Putzig I, Zedler M, Ohlmeier M. [Internet dependency as a symptom of depressive mood disorders (German)]. Psychiatrische Praxis. 2007; 34(S 3):318-22. doi: $10.1055 / \mathrm{s}-2007-970973$

[24] De Berardis D, D' Albenzio A, Gambi F, Sepede G, Valchera A, Conti CM, et al. Alexithymia and its relationships with dissociative experiences and internet addiction in a nonclinical Sample. CyberPsychology \& Behavior. 2009; 12(1):67-9. doi: $10.1089 / \mathrm{cpb} .2008 .0108$

[25] Bahri N, SadeghMoghadam L, Khodadost L, Mohammadzade J, Banafsheh E. Internet addiction status and its relation with students' general health at Gonabad Medical University. Modern Care Journal. 2011; 8(3):166-73.

[26] Nastizai N. The relationship between general health and internet addiction. Zahedan Journal of Research in Medical Sciences. 2009; 11(1):0-

[27] Ghanbari S, Amani A, Pezhman MN, Bidi F, Kareshki H. [Structural analysis of relationship of internet addiction with depression, social adjustment and self-esteem (Persian)]. Scientific Journal of Hamadan University of Medical Sciences. 2012; 19(3):41-8.

[28] Akin A, Iskender M. Internet addiction and depression, anxiety and stress. International online journal of educational sciences. 2011; 3(1):138-48.

[29] Kim K, Ryu E, Chon MY, Yeun EJ, Choi SY, Seo JS, et al. Internet addiction in Korean adolescents and its relation to depression and suicidal ideation: A questionnaire survey. International Journal of Nursing Studies. 2006; 43(2):185-92. doi: 10.1016/j.ijnurstu.2005.02.005

[30] Oh WO. Factors influencing internet addiction tendency among middle school students in Gyeong-Buk Area. Journal of Korean Academy of Nursing. 2003; 33(8):1135. doi: 10.4040/jkan.2003.33.8.1135
[31] Masoudnia E. [Internet addiction and risk of sleep disorder among adolescents (Persian)]. Journal of Research in Behavioural Sciences. 2012; 10(5):350-62.

[32] Janocha A, Klimatskaya LG. Internet addiction disorder in pupils and students of Krasnoyarsk (Russia) and Wroclaw (Poland). Hygeia Public Health. 2011; 46(4):449-52.

[33] Derogatis LR. Brief symptom inventory: Administration, scoring, and procedures manual. New Jersey: National Computer Systems (NCS); 1993.

[34] Dobson KS, Mohammadkhani P. A randomized clinical trial of MBCT, CBT and treatment as usual in the prevention of depresion relapse. Paper Presented at: The World Congress of Behavioural and Cognitive Therapy. 11-14 July 2007; Barcelona, Spain.

[35] Mohammadi A, Naghdi A, Aliverdinia A, Kiani M. [A sociological study of the relationship between social capital and addiction to the internet among the youth in the City of Hamedan (Persian)]. Youths, Culture and Society Research. 2012; 5:1-27.

[36] Tamanaeifar MR, Arfeei FS, Gandomi Z. Relationship between internet addiction with neuroticism in high school students, Kashan, Iran. Bimonthly Journal of Hormozgan University of Medical Sciences. 2014; 17(1):69-75.

[37] Lashgarara B, Taghavi Shahri M, Maheri AB, Sadeghi R Internet addiction and general health of dormitory students of Tehran University of Medical Sciences in 2010. Journal of School of Public Health and Institute of Public Health Research. 2012; 10(1):67-76.

[38] Morahan-Martin J, Schumacher P. Incidence and correlates of pathological Internet use among college students. Computers in human behavior. 2000; 16(1):13-29. doi: 10.1016/s07475632(99)00049-7

[39] Sadat Ahmadi H, Muhammadi Zade F, Ma'soumbeigi $\mathrm{M}$, Sohrabi F. [Prevalence of internet addiction and its relationship with demographic characteristics among Allameh Tabataba'i University students (Persian)]. Educational Psychology. 2012; 8(25):20-30.

[40] Jafari N, Fatehizadeh M. [Investigation of the relationship between internet addiction and depression, anxiety, stress and social phobia among students in Isfahan University (Persian)]. Scientific Journal of Kurdistan University of Medical Sciences. 2012; 17(4):1-9. 